\title{
Scapular dyskinesis: biomechanics, evaluation and treatment
}

\begin{abstract}
The scapular waist comprises the scapula and clavicle and together with the humerus and the external constitute the shoulder, a joint formed by 5 joints. When the scapula moves coordinately the humerus maintaining the axis of rotation of the humerus is called the scapulohumeral rhythm. Scapular dyskinesia are changes in position and scapular movements that predispose to shoulder injuries. Several factors can create changes in the position of the scapula and can be divided into three categories of dyskinetic patterns, which correspond to the three planes of motion on the thoracic, identifying the type of abnormal scapular movement: type I, type II and type III. The objective of this study was to review the pathology and treatment of Scapular Dyskinesia. For this, a bibliographic review study was carried out, which constitutes an important tool to update on a defined theme. For data collection, publications on the topic were used. The literature review was performed in the Bireme databases, and the database Medline, Scielo, and Lilacs were consulted. The keywords were: Scapular Waist, Shoulder, Scapular Movement, Dyskinesia. It is concluded that in rehabilitation of the shoulder, restoration of the normal function of the scapula is of paramount importance, and the synchronization of forces between the scapulothoracic and scapulo-ovarian muscles should be included in rehabilitation, emphasize the strengthening of shoulder depressor muscles, scapular stabilizers, and which act primarily on the shoulder.
\end{abstract}

Keywords: scapular waist, shoulder, scapular movement, dyskinesia
Volume 3 Issue 6 - 2018

Hugo Machado Sanchez, Eliane Gouveia de
Morais Sanchez
Researcher, professor Full, Universidade Federal de Goias, Braz

Correspondence: Hugo Machado Sanchez, researcher professor Full, Universidade Federal de Goias, Rua Dom Emanuel 216, Jataí-GO, CEP 75800-104, Tel+556498I228I36, Email hmsfesio@yahoo.com.br

Received: October 26, 2018 | Published: November 28, 2018

\section{Introduction}

The scapular waist comprises the scapula, clavicle, humerus and outer, together constitute the shoulder, a joint formed by 5 joints. Scapular girdle movements can be described as scapula movements: abduction (prostration), adduction (retraction), upward rotation, downward rotation, elevation and depression. ${ }^{1}$ In order for the shoulder complex to reach its full function, a synergy of joint movements is indispensable, so the scapuloumeral rhythm must be intact, so that if this does not occur, the soft tissues of this complex are subject to overload articular and therefore to diverse lesions. ${ }^{2}$ Therefore, the current review aimed to review the Scapular Dyskinesia, from the point of view of pathology, evaluation and, finally, the physiotherapeutic treatment.

\section{Scapulothoracic biomechanics}

The scapulotracheal joint is a joint considered to be false because it does not present the conventional components of a synovial joint. However, because of its great importance for the shoulder complex, it is widely studied. The movements of this joint are varied and occur by the fascial slip in two spaces: the first occurs between the fascia of the anterior serratus muscle and the subscapularis muscle, and between the fascia of the anterior serratus muscle and the external intercostal muscles. $^{3}$

The movement of elevation of the scapula is approximately $60^{\circ}$, depression from a resting position, only 5 to $10^{\circ}$ can be achieved, this movement is important, as it stabilizes the scapula and elevates the shoulder and the body. the retraction is approximately $25^{\circ}$, the upward and downward rotation is approximately $60^{\circ}$. Abduction of the shoulder in the plane of the scapula occurs 30 to $40^{\circ}$ anterior to the frontal plane. ${ }^{4}$
In the Escapulothoracic (ET) joint, the movements of the scapula require sliding of the scapula over the thorax. There is usually considerable flexibility of soft tissue, which allows movements of the upper limb. ${ }^{5}$

In the sternoclavicular joint (EC) the scapula movements are: elevation, depression, protraction (abduction) and retraction (adduction); in the EC joint: up and down rotation, acromioclavicular joint $(\mathrm{AC})$ : rotation usually with humerus movements: rotation of the upper scapula is a necessary movement for full range of flexion and abduction of the humerus. ${ }^{5}$

According to Kibler, ${ }^{6}$ Kibler, ${ }^{7}$ Greve et al. ${ }^{8}$ The scapulo-humeral rhythm is when the scapula moves coordinately with the humeral head keeping the axis of rotation of the humerus. It can also be considered as the relation of the elevation of the humerus on the scapula, in the movements of elevation of the shoulder, and has the joint action of the glenohumeral and scapulothoracic joints, being less evident in the first $30^{\circ}$, such relation of elevation of the humerus on the scapula generally ranges from 1.5:1 to 2:1. The glenohumeral joint contributes 90 to 120 degrees for abduction of the shoulder, depending on the position of internal or external rotation of the humerus. The remaining $60^{\circ}$ are provided by the elevation of the scapula, this combined movement between the scapula and the humerus is known as the escapuloumeral rhythm. During the first $30^{\circ}$ of glenohumeral abduction, the contribution of scapular elevation is irrelevant, as well as not coordinated with humerus. It is known as the accommodation phase, during which the scapula seeks a position of stability on the chest wall relative to the humerus. ${ }^{9}$

\section{Scapular dyskinesis}

According to Myers et al. ${ }^{10}$ Scapular dyskinesia are changes in the 
position and scapular movements that predispose to shoulder injuries, as they modify the relationship of strength and tension of muscles and affect their efficiency. Scapular dyskinesia may be a consequence of many factors such as biomechanical and physiological abnormalities, bone anatomy altered by posture or bone lesions, muscle injuries due to direct trauma, microtraumas that lead to imbalances, fatigue and pain.

Proper movement of the scapula is considered crucial for normal shoulder function. It serves as a stable base for upper limb function through the glenohumeral joint, but, on the other hand, should allow a large range of shoulder movement. ${ }^{11}$ The efficiency in the generation and transfer of force by the shoulder requires an adequate positioning of the scapula to minimize the loads in the glenohumeral and acromioclavicular joints. ${ }^{12}$

Scapulothoracic dysfunction has recently been recognized as an important factor in the diagnosis and treatment of many common shoulder injuries. ${ }^{12}$ Due to its importance in the shoulder complex, abnormal movement of the scapula has been associated with pathologies such as impact syndrome, frozen shoulder and glenohumeral instability. ${ }^{13}$

Warner et al. ${ }^{14}$ evaluated the scapular asymmetry in patients with glenohumeral instability and impact syndrome through a topographic analysis and found an association between scapulothoracic dysfunction and lesions that generate clinical dysfunction in the shoulder.

The question that has not been answered and remains to this day is whether this dysfunction represents a primary or secondary phenomenon in shoulder injuries. Due to discussions like this, the more detailed understanding of the scapulothoracic movement has been growing considerably. ${ }^{15,16}$

Several factors can create changes in the position of the scapula such as poor posture, excessive rest posture, thoracic and cervical kyphosis, lordosis that can result in excessive scapular and acromial protraction, clavicle fractures, acromioclavicular joint injuries, instabilities, osteoarthritis, changes in the function of the muscles that control the scapula, lesions along the long thoracic nerve (which may alter the muscle function of the anterior serratus), spinal nerve injuries (may alter trapezius muscle function, causing abnormality in stabilization, which occurs in approximately $5 \%$ of cases). Generally the muscles that stabilize the scapula are directly related to directtrauma trauma, microtrauma, leading to muscle weakness and inhibition of movement by painful conditions around the shoulder. The anterior serratus and trapezius are the most susceptible to the effects of inhibition, and are most often involved in the early stages of shoulder pathology. ${ }^{17}$

According to Borloz et al. ${ }^{2}$ joint, ligament or muscle problems near the scapula can lead to poor positioning or an altered movement pattern. This abnormal condition is called scapular dyskinesia, which appears to be a nonspecific response to a painful shoulder condition.

According to Kisner et al..$^{5}$ and Borloz et al. ${ }^{2}$ a defective scapular posture, caused by muscular imbalances, also generates imbalances of length and muscular strength in the umeral muscles, altering the mechanics of the glenohumeral joint. A forward bending of the scapula is associated with a retracted minor pectoral muscle and possibly anterior serratus weakness or trapezius. This scapular posture alters the humeral posture on the glenoid, assuming a relatively abducted and internally rotated position, resulting in retracted gleno-umeral internal rotators and elongated or weak lateral rotators.
Muscle imbalance is the difference in strength and flexibility between muscle groups acting on the same joint, that is, when a given muscle group is stronger and/or more stressed than its respective antagonist. ${ }^{18-20}$

The imbalance can be a causal factor or be associated with several factors, such as: improper use, excessive repetition, poor posture, antalgic posture, joint pathologies, muscular pathologies, contractures or adhesions, neurological deficits, disuse or atrophy, indiscriminate practice of sports activities, among others. ${ }^{21}$

The scapulothoracic pathology occurs in chronic painful conditions in which the glenohumeral movement has been gradually reduced or in which the glenohumeral joint has been immobilized for a long period. In most circumstances, the scapulothoracic joint becomes a secondary problem. Increased shoulder pain may result in muscle spasm in the supraspinatus, trapezius, rhomboids, large dorsal and subscapularis. In the event that a scapulothoracic joint does not function properly, the individual will be able to achieve only 100 to $120^{\circ}$ of passive abduction, and abductionpassive, and active abduction may be even more limited.

The effects of scapular dyskinesia are the loss of retraction that causes the loss of stability at the time of raising the arm and the lack of scapular protraction around the chest wall increases the deceleration of the forces at the shoulder joint, causes changes in the normal conditions of the relationship between the glenoid and the humerus in addition to creating a functional glenoid antivenom, which may increase tensions over the anterior stabilizers, glenoid lip structures and glenohumeral ligaments, thus increasing the risk of injury. The loss of the ability to raise the acromion may be a secondary source of shoulder impact on other problems, such as glenohumeral instability. The anterior serratus and trapezius lower fibers are the first muscles involved in inhibiting muscle dysfunction. It can be seen at the beginning of many other shoulder problems, such as rotator cuff tendonitis and glenohumeral instability. This may play a role in the development of new symptoms. If scapular movement is impaired, forces generated from the lower extremity and trunk will not be effective to transmit the upper extremity. ${ }^{17}$

In addition, inhibition of muscles resulting from scapular dyskinesia is a nonspecific response to a painful shoulder condition, rather than a specific response to a glenohumeral pathology, $68 \%$ of patients with rotator cuff abnormalities, $94 \%$ in the glenoid lip and $100 \%$ with glenohumeral instabilities. Inhibition is seen as a diminished ability of the muscles to exert torque and stabilize the scapula as well as disorganization of the muscles around the shoulder. The exact nature of this inhibition is unclear. A proprioceptive motor pattern, based on direct or indirect muscle injury, fatigue or muscle tension is suggested to alter the activation of muscle spindles, contractures or shortening of muscles and ligaments around the shoulder, affecting the position and mobility of the scapula. ${ }^{17}$

The relationship of dyskinesia with another type of injury, in the case of acromioclavicular joint injury, was analyzed in the study by Murena et al. ${ }^{22}$ who found that in cases of acromioclavicular lesions (type III dislocations), scapular dyskinesia can be avoided through the surgical treatment of this lesion, this result was obtained after the observation of 34 patients.

\section{Scapular dyskinesisevaluation}

In rehabilitation of the shoulder, in order to have a correct indication and execution of rehabilitation, there is a need not only for knowledge 
of the pathology, but also for its biomechanical/functional imbalance, with adequate anamnesis, physical examination and complementary exams. $^{8}$

Scapular assessment should include normal scapular movements, assessment of movement dynamics, muscle activation, and corrective maneuvers should be made. Lumbar lordosis, pelvic tilt and hip rotation should be checked. The thoracic and cervical posture should also be evaluated, since the increase in thoracic kyphosis and the presence of scoliosis may have a direct effect on the movement of the scapula, creating an abnormal contour of the scapular movement. Excess cervical lordosis can affect scapular retraction and protraction. Evaluation of the scapula by itself should be done mainly from the posterior aspect. ${ }^{17}$

The musculature of the scapulothoracic joint should be evaluated in order to verify the presence of spasm in the rhomboid, large dorsal, upper and lower trapezius, subscapular, minor round, infra-spinal and supraspinal muscles. These areas should also be evaluated for active trigger points. The evaluation of trigger points in these muscles can radiate pain to the middle deltoid and elbow bundles and, in severe cases, through the arm. ${ }^{9}$

Muscle weakness from scapular dyskinesia is more common in the lowering arm. An effective maneuver for the evaluation of scapular muscle strength is to perform an isometric contraction of the scapula in retraction. The scapular muscle weakness can manifest as a burn-like pain in less than 15 seconds, while the normal scapula generates a contraction at this position for 15 to 20 seconds without pain or muscle weakness. Wall push-ups are effective in assessing the muscle strength of the anterior serratus. Scapular abnormalities can be observed between 5-10 flexions. ${ }^{17}$

The scapular position can be evaluated in several ways. Abnormalities of elevation and rotation should be analyzed in the resting position. Weakness of the anterior serratus muscle will create a super-medial prominence of the scapula and depression of the acromion, while weakness of the trapezius muscle will create a lower prominence and elevation of the acromion. There may be pain at the anterior palpation over the shoulder in the coracoid process, secondary to the attempt of the minor pectoralis muscle and short head of the biceps to adapt. The super-medial part (upper angle) can be painful on palpation or with movement because of the scapula and trapezius lift. Movement and scapular position should be analyzed both in the phases of the movement of the shoulder and in the shoulder depression. ${ }^{17}$

Some tests are described by Kibler et al. ${ }^{17}$ to assess scapular dyskinesia, they are:

1. Scapular assistance test (Figure 1): evaluates the impact of the scapula and the involvement with the glenoid. This procedure evaluates the activity of the anterior serratus and inferior trapezius muscles. Elimination or modification of the symptoms of these muscles indicates the focus of rehabilitation;

2. Scapular retraction test (Figure 2): Manually wraps the scapula stabilizer in a closed position over the thorax. This position gives a stable base of origin to the rotator cuff and will often improve the strength of the rotator cuff. The scapular retraction test also demonstrates the impact of the scapula and the involvement with the glenoid;

3. Slip test of the lateral scapula: it is more sensitive to dyskinetic patterns, because excessive protraction occurs. A $1.5 \mathrm{~cm}$ asymmetry is the limit of abnormality and is most commonly seen in abduction of the shoulders at $90^{\circ}$. In the first position, the patient has relaxed arms at the side of the body (Figure $3 \mathrm{~A})$. In this position, the lower-medial angle of the scapula is palpated and marked on both sides. The reference point is on the backbone is the next thorny process. The reference measurements from the spine to the medial aspect of the scapula are measured on both sides. The same is done with arms equal to or less than $90^{\circ}$ abduction of the arm with maximum internal rotation of the glenohumeral joint (Figure 3B).

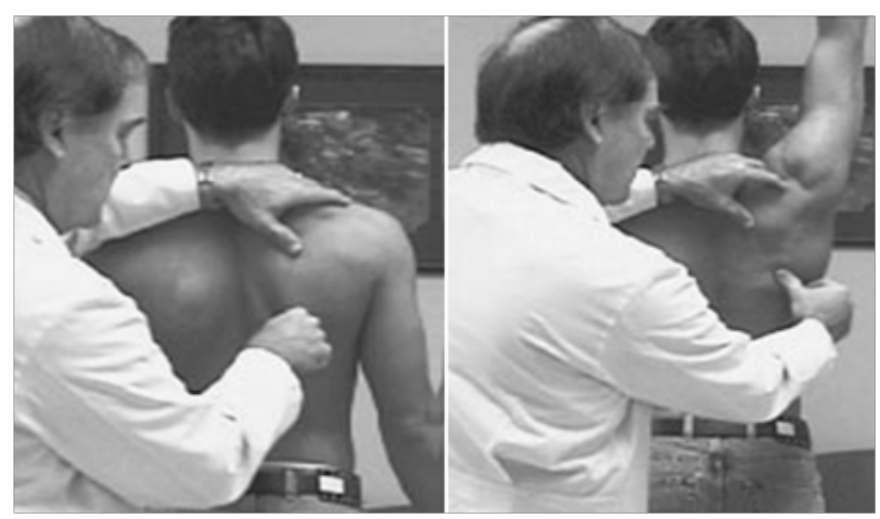

Figure I The examiner observes the activity of the anterior serratus muscle and the inferior trapezius when the arm is raised. When there is relief of symptoms the test is positive. The examiner with his right hand at the position at the beginning of the test.Afterwards elevation of the arm, scapular rotation.

Source: kibler et al. ${ }^{17}$

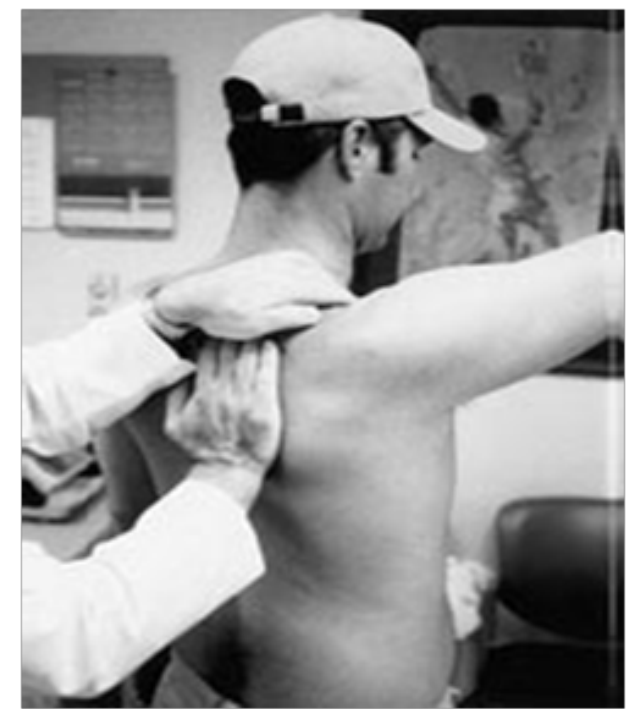

Figure 2 Scapular retraction test. The examiner stabilizes the scapula medially and the arm is raised or rotated externally. When there is relief of symptoms, the test is positive.

Source: Kibler et al. ${ }^{29}$

A more objective and accurate but much more complex way to assess the movements of the scapula is the three-dimensional biomechanical analysis that is able to show that the scapula normally moves around three axes of movement simultaneously in the common day-to- Day 11]. It is important to emphasize that in this type of analysis, in which it is necessary to place markers on the scapula, there is the difficulty to trace the movement of this because a significant slip of the scapula occurs in relation to the skin. ${ }^{23}$ 


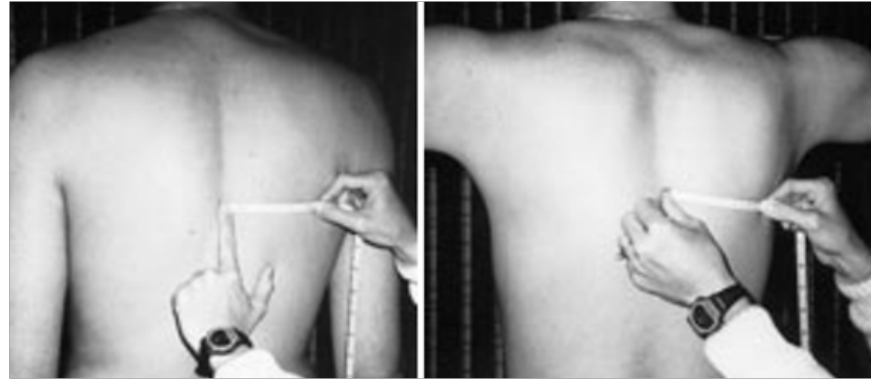

Figure 3 A - Position: arms at rest; B - Position: both arms abducted at $90^{\circ}$ with maximum internal rotation. Source: Kibler et al. ${ }^{17}$

In this way, some methods have been developed for a better accuracy of the scapula movement analysis. The insertion of intracortical pins associated with an electromagnetic device is obviously the most accurate, but it is an invasive and clinically unfeasible method. ${ }^{11}$

Other non-invasive methods, based on optical or electromagnetic tracking devices, have been developed to analyze the movement of the scapula and used for diagnostic and evaluation purposes. ${ }^{24}$

From the previously mentioned methods, a method using a cluster positioned in the acromion based on retro-reflective markers was developed and validated to track the three-dimensional movement of the scapula. ${ }^{25}$ This method, clinically feasible, can aid in the understanding of the movements of the scapula for the global movement of the shoulder. In this way, it can generate benefits both in the clinical area and during a physical exam, in a surgical reconstruction or in shoulder rehabilitation programs..$^{13}$

Digital biophotogrammetry has been considered as an alternative for the quantitative evaluation of postural asymmetries in postural evaluation, and can be used to perform linear and angular measurements. ${ }^{26,27}$

In the studies of Juul-Kristensen et $a .^{28}$ it was found that the dyskinesia scapula presented greater medial mapal misalignment, a greater horizontal distance from the lower scapular angle and greater passive internal rotation of the shoulder, and also verified that cases with a higher degree of scapular dyskinesia presented a reduction in the capacity of work and health in general, and also noticed that upper trapezius myalgia is related to scapular dyskinesia.

\section{Classification of scapular dyskinesis}

According to Kibler $^{7}$ \& Kibler et al. ${ }^{29}$ the three-dimensional biomechanical analysis of scapular movements shows that the scapula moves around three axes of movement simultaneously. Abnormal patterns of movement in scapular dyskinesia are best observed by first determining the position of the scapula with the patient's arms resting on the side, noting the movement of the scapula when the arms are raised and lowered in the scapular plane. They can be divided into three categories of dyskinetic patterns, which correspond to the three planes of motion on the thorax, and identify the type of abnormal scapular movement and thus aid rehabilitation and recovery of flexibility required by the muscle.

Kibler $^{7}$ classifies scapular dyskinesia in 3 types, they are:

1. Type I (Figure 4A) is characterized by the inferior and medial prominence (lower angle) of the scapula. This movement is abnormal in rotation about a transverse axis;

2. Type II (Figure 4B) is characterized by representing abnormal rotation about a vertical axis, of all scapula medially;
3. Type III (Figure 4C) is characterized by the prominence of the upper and medial scapula border.

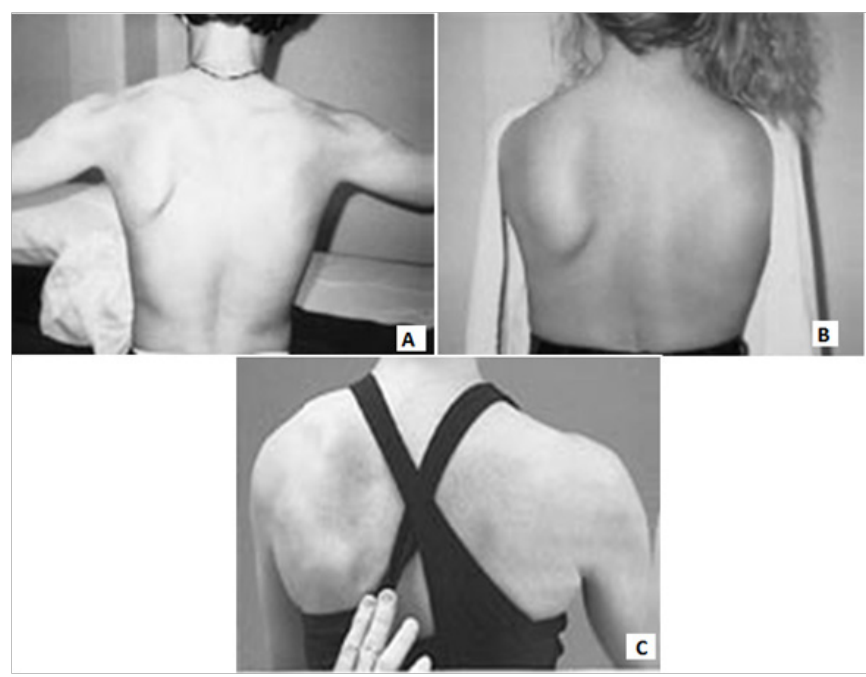

Figure $4 \mathrm{~A}$ - Type I, with emphasis on the lower and medial part of the left scapula; Type II, with prominence of all the medial part of the left scapula; C - Type III, with prominence of the upper and medial part of the left scapula.

Source: Kibler et al. ${ }^{17}$

\section{Treatment}

Most scapular abnormalities can be treated by Physical Therapy to relieve the symptoms associated with stiffness to restore the pattern of muscle strength and activation. Surgical treatment is used to repair the source of abnormalities and is often an integral part of the treatment program. ${ }^{17}$

The treatment of muscular imbalances consists in promoting a rebalancing of the muscular chains, lengthening what is shortened and strengthening what is weak. ${ }^{30,31}$

In shoulder rehabilitation, restoration of the normal function of the scapula is of paramount importance and the synchronization of forces between the scapulothoracic and scapulo-ovarian muscles should be included in rehabilitation, emphasizing the strengthening of the depressor muscles of the shoulder (subscapular, infraescapular and minor round), scapular stabilizers (trapezius upper and lower fibers, anterior serratus and rhomboids) and those that act primarily on the shoulder (deltoid, pectoralis major and dorsal). The anterior serratus and trapezius muscles contribute to a good positioning of the scapula during arm elevation and synergist activities when there is deltoid muscle action and are essential for the movement of the scapula over the rib cage, especially in conjunction with the arm elevation. ${ }^{32}$

Scapular rehabilitation will address muscle retractions and imbalances and restore good dynamic stability, starting with analytic work evolving into more functional and specific exercises. ${ }^{2}$

The muscular strength and resistance of the stabilizing musculature of the scapula are important to maintain a correct joint arthrokinematics. The function of the scapulothoracic joint and surrounding musculature is important in maintaining a functioning shoulder. Muscle spasm, weakness, and poor neuromuscular coordination of the stabilizing muscles directly affect the glenohumeral movement. In case the scapula does not move properly over the rib cage, nor maintain the correct relationship between muscle length and tension, or correctly orient the glenoid fossa with the humeral head, the asynchronous movement in the shoulder complex will eventually produce injury. 
Thus, the stabilizers of the scapula can and should be strengthened, requesting the individual to perform surveys, horizontal abduction in the ventral decubitus, scapular retraction exercises and maneuvers for neuromuscular control. ${ }^{9}$

Before treating a reduction of glenohumeral movement, the scapulothoracic motion must be restored. To reduce muscle spasms, it is necessary to use moist heat, ultrasound, mobilization of soft tissues, release of trigger points and scapular mobilization. ${ }^{9}$

In a study by Brum et al. ${ }^{33}$ with the aim of evaluating and comparing the electromyographic activity of muscles of the shoulder girdle and arm in healthy volunteers in wall-press $90^{\circ}$, wall-press $45^{\circ}$, benchpress and push- fixed - axial load (EFCA). The wall-press $90^{\circ}$, wallpress $45^{\circ}$, bench-press and push-up exercises, classified as EFCA, were performed isometric on a stable surface. The $90^{\circ}$ wall-press exercise was performed with the volunteer in the orthostatic position, the upper limb dominant at $90^{\circ}$ of shoulder flexion in the sagittal plane and wrist at $90^{\circ}$ extension. The wall-press $45^{\circ}$ was performed with the same positioning of the wall-press $90^{\circ}$, changing only the angle of flexion of the shoulder to $45^{\circ}$. To perform the bench-press exercise, the volunteer was placed in the supine position, with the feet resting on the stretcher, upper limb dominant at $90^{\circ}$ of shoulder flexion and $90^{\circ}$ of wrist extension. Finally, the push-up exercise was performed with the volunteer in the supine position, supported on the feet, neutral position of trunk and with the dominant upper limb in $90^{\circ}$ of flexion of shoulder and wrist in $90^{\circ}$ of extension. It can be observed that the exercises studied produced low electromyographic activity in the biceps brachii, not being the most indicated to activate this muscle. However, the push-up and bench-press exercises significantly activated the anterior fibers of the deltoid muscle and the anterior serratus and the wall-press exercises $90^{\circ}$ and wall-press $45^{\circ}$ significantly activated the superior fibers of the trapezius muscle, can be used in clinical practice to activate this musculature.

According to Barbosa et al..$^{32}$ good rehabilitation of patients with scapular dysfunction should use exercises that do not attack the joint in the first stages of rehabilitation, such as wall push up and passive and active exercises, for a good contraction of the rotator cuff muscles, the anterior serratus and trapezius upper fibers. Evolving into the next stages of rehabilitation with higher overload exercises like knee push up, scaption, elbow push up for better synchronization of scapular muscles and, in the last phase to perform plyometric and proprioceptive work to better return the patient to his activity, whether it is work or sports.

According to Kibler \& McMullen, ${ }^{17}$ the rehabilitation of scapular dyskinesia is based on a protocol from proximal to distal, which emphasizes movement and scapular coordination, and complementary trunk and hip movements, as follows:

In the acute phase (usually 0 to 3 weeks) (1) Initially, avoid movements and painful positions of the arm and establish the facilitation of scapular movement; (2) Mobilization of soft tissues and muscle stretching of the minor pectoral muscles, levator scapula, superior trapezius, large dorsal and infra-spiny if there is limitation of movement. Stretching techniques in the restoration of muscle flexibility are: active, active-assisted stretching, passive and proprioceptive neuromuscular facilitation, are effective in restoring muscle flexibility as well as in the range of motion of the glenohumeral joint; (3) Start from the upper end by shifting the weight, swinging the exercises. The exercises should be rhythmic with the stabilizing ball and weight support to promote isometric contraction. Use closed-loop kinetic (CCF) exercises where the hand is supported or has weight applied to it, at different planes and altitude levels if scapular positioning is adequate. These can be initiated at low levels of abduction and external rotation, then can progress to $90^{\circ}$ of abduction until tolerated for the patient; (4) Started scapular movement exercises without arm elevation. Use flexion and medial rotation of the trunk to facilitate scapular protraction. Use active lateral extension and rotation of the trunk, hip extension to facilitate scapular retraction; (5) Include movement of the arm with exercise of movement of the scapula to promote the improvement of the scapular mobility. Initially, keep the arms close to the body to minimize the intrinsic load. An excellent start of exercise for scapular stabilization is the low row, which includes trunk/hip extension, scapula retraction, and arm extension;

In the recovery phase ( 3 to 8 weeks) the activation of proximal and muscular stability is necessary for proper movement and strengthening of the scapula. The force depends on the movement, and the movement depends on the posture; (1) Start with larger load exercises in CCF such as wall push-ups, table push-ups and modified prone push-ups. In addition, increasing the level of CCF elevation exercises improves scapular control. The patient's position in TLC, exercise for hand placement, then moves the body relative to the fixation of the hand, to define the plane and degree of elevation; (2) Add elevation and rotation of the arm to the standard scapula movement exercises, up to the level tolerated by the patient. If intrinsic loads are very large with the introduction of active lifting, active axial movement exercises are used, in which the hand is freely movable, wall-slide is an example of exercise; (3) Hip and trunk extension exercises with scapular retraction, and hip and trunk flexion with scapular protraction. Pull at different angles and planes of motion. They are used to reproduce the proper functions of the scapula;

-Maintenance phase (6 to 10 weeks), occurs (1) When there is good control of scapular movement throughout the shoulder elevation, it will initiate plyometric exercises; (2) Overhead dumbbell presses and dumbbell punches are exercises that require good muscle control complete range of glenohumeral movement.

In a rehabilitation protocol developed by Lazáro et al., ${ }^{34}$ in order to improve the pain, recovery of range of motion and muscle strength, resources such as electrothermotherapy and kinesiotherapy were used. The protocol was divided into 4 phases, with different goals and behaviors:

1. Phase I: reduce the inflammatory process, relieve pain and swelling. At this stage the resources used were ultrasound, short waves, pendant exercises of Codman, with dumbbells of $1 \mathrm{~kg}$, and active stretching of supraspinatus;

2. Phase II: slow muscle atrophy and maintain or increase flexibility. In this phase were added the exercises of WMD, with stick, and isometric strengthening;

3. Phase III: restore painless range of motion and muscle strengthening. At this stage the devices were used, electrotherapeutics, if necessary, and strengthening exercises were added;

4. Phase IV: to improve muscular performance, functional recovery and proprioception. In this last phase, the proprioceptive exercises were included, rubber ball throw in the elastic bed and the position of cat with support of the hands in the ball type bean. 
The importance of the strengthening of scapular stabilizers was studied by Başkurt et al. ${ }^{35}$ in which the researchers evaluated 40 middle-aged subjects (men and women) for 6 weeks with a frequency of 3 times a week and found after this period that the potentiation of scapular stabilizers was more effective in reducing pain in the shoulder, originating from Impact Syndrome, than non-specific stretching and strengthening for the scapular stabilizing muscles. According to the authors, such findings are justified by the fact that scapular stabilization repositions the scapula and decreases the degree of scapular dyskinesia.

\section{Conclusion}

Based on the bibliographical studies, it can be concluded that, in order to obtain the synchronism of forces between the scapulothoracic and scapulo-ovarian muscles, the strengthening of the shoulder depressor muscles (subscapular, infraescapular and minor round), scapular stabilizers (upper and lower inferior, anterior serratus and rhomboids) and those that act primarily on the shoulder (deltoid, pectoralis major and dorsal). The present study specified some exercises that is performed for the overall treatment of the shoulder and scapular girdle joint. There were several limitations in the study, due to the difficulty of finding articles on scapular dyskinesia, the types and treatments indicated.

\section{Acknowledgment}

None.

\section{Conflicts of interest}

The authors declare no conflicts of interest.

\section{References}

1. Thompson CW, Floyd RT. Manual de cinesiologia estrutural. 14th edn São Paulo: Manole; 2002:279.

2. Borloz S, Graf V, Gard S, et al. Scapulardyskinesis. Rev Med Suisse. 2012;19;8(367):2422-2428.

3. Kapandji IA. Fisiologia articular esquemas comentados de mecânica humana. 5th edn. São Paulo: Manole. 2001;(1):255.

4. Smith LK, Weiss EL, Lehmkuhl LD. CinesiologiaClinica de Brunnstrom. 5th edn. São Paulo: Manole. 1997;538

5. Kisner C, Colby LA. Exercícios Terapêuticos- Fundamentos e Técnicas. 3rd edn. São Paulo: Manole; 2009.

6. Kibler WB. Role of the scapula in the overhead throwing motion. ContempOrthop. 1991;22:525-532.

7. Kibler WB. The role of the scapula in athletic shoulder function. Am J Sports Med. 1998;26:325-237.

8. Greve JMD, Amatuzzi MM. Medicina de reabilitação aplicada à ortopedia e traumatologia. São Paulo: Roca. 1999;444.

9. Andrews JR, Harrelson GL, Wilk KE. Reabilitação física das lesões desportiva. 2nd edn. Rio de Janeiro: Guanabara Koogan. 2000;504.

10. Myers JB, Laudner KG, Pasquale MR, et al. Scapular position and orientation in thowing athletes. Am J Sports Med. 2005;33(2):263-271.

11. McClure PW, Michener LA, Sennett BJ, et al. Direct 3-dimensional measurement of scapular kinematics during dynamic movements in vivo. J Shoulder Elbow Surg. 2001;10(3):269-267.

12. Dome DC, Kibler WB. Evaluation and management of scapulothoracic disorders. Current Opinion in Orthopaedics. 2006; 17(4):321-324.
13. Van AC, Van HK, Eversdijk M, et al. Recording scapular motion using an acromion marker cluster. Gait \& Posture. 2009;29(1):123-128.

14. Warner JJ, Micheli LJ, Arslanian LE, et al. Scapulothoracic motion in normal shoulders and shoulders with glenohumeral instability and impingement syndrome: a study using moireopographic analysis. Clinical Orthopaedics and Related Research. 1992;(285):191-199.

15. Amasay T, Karduna AR. Scapular kinematics in constrained and functional upper extremity movements. Journal of orthopaedic and sports physical therapy. 2009;39(8):618-627.

16. Reinold JF, Scamilla R, Wilk KE. Current concepts in the scientific and clinical rationale behind exercises for glenohumeral and scapulothoracic musculature. Journal of Orthopaedic and Sports Physical Therapy. 2009;39(2):105-117.

17. Kibler WB, Mcmullen JATC. Scapular dyskinesis and its relation to shoulder pain. Journal of the American Academy of Orthopaedic Surgeons. 2003;11(2):142-151.

18. Liebenson C, Lardner R. Identification and treatment of muscular chains Ynamic Chiropratic. 1999;17(18):1-9.

19. Kollmitzer J, Ebenbichler GR, Sabo A, et al. Effects of back extensor strength training versus balance training on postural control. Medicine Science in Sports and Exercise. 2000;32(10):1770-1776.

20. Klee A, Jollenbeck T, Wiemann K. Correlation between muscular function and posture -lowering the degree of pelvic inclination with exercise. International Society of Biomechanics in Sports. 2004.

21. Stokes M. Neurologia para fisioterapeutas. São Paulo: Premier 2000:154-170.

22. Murena L, Canton G, Vulcano E, et al. Scapular dyskinesis and SICK scapula syndrome following surgical treatment of type III acute acromioclavicular dislocations. Knee Surg Sports TraumatolArthrosc. 2013;21(5):1146-1150.

23. Karduna AR, Mcclure PW, Michener LA, et al. Dynamic measurements of three-dimensional scapular kinematics: a validation study. Journal of Biomechanical Engineering. 2001;123(2):184-190.

24. Meskers CG, Van de Sande MA, De Groot JH. Comparison between tripod and skin-fixed recording of scapular motion. Journal of Biomechanics. 2007;40(4):941-946.

25. Salvia P, Jan SV, Crouan A, et al. Precision of shoulder anatomical landmark calibration by two approaches: A CAST-like protocol and a new anatomical palpator method. Gait \& Posture. 2009;29(4):587-591.

26. Watson AWS. Procedure for the production of high quality photographs suitable for the recording and evaluation of posture. Revista de Fisioterapia da Universidade de São Paulo. 1998;5(1):20-26.

27. Iunes DH, Castro FA, Salgado HS, et al. Confiabilidade intra e interexaminadores e repetibilidade da avaliação postural pela fotogrametria. Revista Brasileira de Fisioterapia. 2005;9(3):327-334.

28. Juul KB, Hilt K, Enoch F, Remvig L, Sjogaard G. Scapular dyskinesis in trapezius myalgia and intraexaminer reproducibility of clinical tests. Physiother Theory Pract. 2011;27(7):492-502.

29. Kibler WB, Uhl TL, Maddux JW, et al. Qualitative clinical evaluation of scapular dysfunction: A reliability study. J Shoulder Elbow Surg. 2002;11(6):550-556.

30. Kollmitzer J, Oddsson L, Ebenbichler GR, et al. Postural controlduring lifting. J Biomech. 2002;35(5):585-594.

31. Kolyniak IEGG, Cavalcanti SMB, Aoka S. Avaliação isocinética da musculatura envolvida na flexão e extensão do tronco: efeito do método pilates. Revista Brasileira de Medicina do Esporte. 2004;10(6):487-490. 
32. Barbosa G, Reis J, Invernici LV, et al. Reabilitação das disfunções escapulares: uma revisão. centro de traumatologia do esporte. Departamento de Ortopedia e Traumatologia. UNIFESP. São Paulo. 2005: 5 .

33. Brum DPC, Carvalho MM, Tucci HT, et al. Avaliação eletromiográfica de músculos da cintura escapular e braço durante a realização de exercícios com extremidade fixa e carga axial. Revista Brasileira de Medicina e Esporte. 2008;14(5):466-471.
34. Lázaro FTO, Bertolini GRF, Nakayama GK. Tratamento fisioterapêutico em pacientes acometidos por lesões e alterações cinésio-funcionais do manguito rotador. Arquivo de Ciências e SaúdeUnipar. 2004;8(1):73-77.

35. Başkurt Z, Başkurt F, Gelecek N, et al. The effectiveness of scapular stabilization exercise in the patients with subacromial impingement syndrome. J Back MusculoskeletRehabil. 2011;24(3):173-179. 